Dr. C. Eisenlohr, Zur Lehre von der acuten spinalen Paralyse. 219

\title{
$\mathrm{XI}$.
}

\section{Zur Lehre von der acuten spinalen Paralyse.}

\author{
Von \\ Dr. C. Eisenlohr, \\ Assistenz-Arzt an der medicinischen' Klinik zu Heidelberg.
}

Die Aufstellung einer neuen Krankheitsgruppe, der paraly sie spinale antérieure aigue de l'adulte durch Duchenne hat in letater Zeit auf bisher wenig beachtete Lähmungsformen die Aufmerksamkeit gelenkt und eine Reihe von casuistischen Mittheilnngen sowohl, als darauf gestützten Reflexionen hervorgernfen. Die Schlussfolgerung Duchenne's, dass die anatomische Ursache der genannten Affection mit derjenigen der spinalen Kinderlähmung identisch sei, gewann durch die von Gombault vorgenommene Untersuchung des Rückenmarks einer Frau, die viele Jahre nach der Attaque der Lähmung an Lebercarcinom zu Grunde gegangen war, eine anatomische Basis. Ausser einer genauen Geschichte seines Falles giebt $\mathrm{Gombault}$ (Archives de Physiologie 1873. Nr. 1) eine Notiz über zwei Beobachtnngen, von denen die eine $\mathrm{Charcot}$, die andere $\mathrm{Cuming}$ angehört.

Letztere war schon früher in Dublin Quaterly Journal 1869 als "Case of extensive paralysis from morbid condition of the spinal cord, probably congestion" von $\mathrm{Cuming}$ selbst veröffentlicht. In einer ausführlichen Brochüre (Considérations sur l'atrophie aiguë des cellules motrices, Paris 1873) bespricht Petitfils die beiden analogen Affectionen der Kinder und Erwachsenen und bringt ebenfalls zwei neue Beobachtungen (von Charcot) bei. Petitfils recurrirt noch auf einen Fall von $\mathrm{Hallopean} \mathrm{(resp.} \mathrm{P.} \mathrm{Lucas} \mathrm{Champion-}$ nière), der in Hallopeau's Studie sur les myélites chroniques diffuses (Archives générales 1871 und 1872 T. XIX. p. 70. 
Observat. G.) unter dieser Bezeichnung erzählt wird - unter Hinweis auf die frappante Analogie mit dem Bilde der spinalen Kinder]ähmung.

In Deutschland haben Bernhardit (Archiv für Psychiatrie und Nervenkrankheiten IV. Band, 2. Heft. 1873) aus Westphal's und Frey aus Kussmaul's Klinik (Berliner Klinische Wochenschrift 1874 Nr. 1, 2, 3) einige einschlägige Fälle publicirt.

Frey betont besonders das Vorkommen von Lähmungen, die in relativ kurzer Zeit wieder vollständig zurückgehen und belegt sie mit dem für analoge Fälle der paralysis infantilis gebräuchlichen Namen temporärer spinaler Lähmungen.

Schon Gombault macht am Schlusse seiner Arbeit auf die Aehnlichkeit aufmerksam, die zwischen der Duchenne'schen Affection und einer seit Landry häufig beschriebenen Lähmungsform, der paralysie ascendante aiguë oder extenso-progressive aiguë besteht. Petitfils widmet der Vergleichung der letzteren mit den acuten Formen spinaler Lähmung bei Kindern und Erwachsenen ein längeres Exposé und kommt zu dem Schlusse, dass die drei Affectionen eine zusammengehörige Gruppe mit demselben anatomischen Substrat, einer acut zu Stande kommenden Atrophie der motorischen Zellen der Vorderhörner bilden. Duchenne selbst bringt die Symptome der Landry'schen paralysie ascendante aiguë mit einer diffusen Myelitis in Zusammenhang, indem er die Störungen der Sensibilität, der Functionen der Blase und des Mastdarmes für ein characteristisches Merkmal derselben hält. Als 3. Species der acuten Atrophie der Vorderhornzellen führt er seine paralysie générale spinale antérieure subaiguë - eine ziemlich langsam und chronisch verlaufende Form - ein.

Ohne auf die berïhrte Classification bei der Lückenbaftigkeit und Vieldentigkeit des anatomischen Nachweises Werth zu legen, möckte ich das Vorkommen gewisser Uebergangsformen betonen, die keinem der etablirten Typen entsprechen, sondern in einzelnen Zügen mehr der paralysie spinale antérieure aiguë de l'adulte, in anderen mehr der acuten progressiven Paralyse sich anschliessen.

Ich theile im Folgenden einen Fall mit, der zweifellos der in Rede stehenden Gruppe von Paralysen angehört und mir gerade den Zusammenhang der verschiedenen Formen zu beleuchten geeignet scheint. Derselbe wurde im verflossenen Sommel auf der Klinik des Herrn Geh. Hofrath Friedreich, dem ich für die Erlaubniss der Publication anfrichtigen Dank sage, ron mir beobachtet. 
Der 33 jährige Kaufmann A. H. liess sich am Abend des 6. Juni 1873 in die medicinische Klinik aufnehmen.

Derselbe hatte sich bisher stets einer guten Gesundheit erfrent; nur um Ostern desselben Jabres will er nach einer Erkältung an einer schmerzlos aufgetretenen Bewegungsstörung in beiden Armen gelitten haben, die nach 1 bis 2 tägiger Dauer spontan wieder verschwunden sei. Die Angaben des Patienten lauteten übrigens so unbestimmt, dass sich die Natur dieser Affection durchaus nicht präcisiren liess. An Syphilis hatte H. nicht gelitten.

Am Morgen des 30. Mai begab sich H. nach einer durchtanzten Nacht bei kühler Temperatur leicht gekleidet nach Hause. Im Laufe der folgenden Tage stellten sich stechende Schmerzen und rasch zunehmende motorische Schwäche in den unteren Extremitäten ein. Ein am 3. Juni genommenes Dampfbad steigerte die Erscheinungen; vom 4. ab liessen zwar die Schmerzen nach, dagegen erreichte die Bewegungsstörung einen solchen Grad, dass Patient weder stehen noch gehen konnte. Subjective Fiebererscheinungen, Kopf- oder Rückenschmerzen, Störungen des Appetits und der Verdauung waren nicht vorhanden, ebensowenig Gefühl von Taubheit oder Ameisenkriechen in der Haut der Beine.

Am Abend des 6. Juni wurde folgender Status notirt.

Der Kranke ist von kräftigem musculösem Bau und sehr gut entwickeltem Panniculus. Die Hauttemperatur erhöht - 39,2, der Puls etwas beschleunigt -84 .

Die oberen Extremitäten sind gut beweglich, doch hat die Energie der Muskeln entschieden abgenommen, Druckkraft der Hände herabgesetzt, im linken Arm Gefühl von Scliwere.

Sehr ausgesprochen ist die Parese beider Unterextremitäten; dieselben können in toto nicht gehoben, die Kniee nur bei aufliegenden Fersen gebeugt werden. Die Bewegungen in den Fussgelenken sind weniger alterirt, Dorsal- und Plantarflexion der Füsse wird mit leidlicher Energie ausgeführt. Im rechten Bein sind die Lähmungserscheinungen etwas stärker ausgesprochen als im linken. Der Kranke kann sich wegen Schwäche der Hüftmusculatur nur mit grösster Mühe im Bett aufrichten. Die Muskeln des Stamms sind intact, die Bewegungen des Halses und Kopfes vollkommen trei. Die Bewegungen der Zunge, der Gaumen- und Schlundmuskeln zeigen keine Functionsstörung, das Zäpfchen steht gerade.

Die Sensibilität ist weder erhöht noch vermindert, Berührung und Druck wird in richtiger Weise percipirt und localisirt. Keinerlei abnorme Sensationen, Schmerzen weder spontan noch bei passiven Bewegungen.

Druck auf die processus spinosi nirgends empfindlich.

Die Reflexerregbarkeit ist in den unteren Extremitäten entschieden gesteigert, bei leiser Berührung der Fusssohle oder des Fussrückens treten sofort fast convulsible Zuckungen in den Adductoren des Oberschenkels, dem tensor fasciae latae und dem Quadriceps ein.

In der Gegend des linken Malleolus internus ist Oedem und leichte Röthe der Haut zu bemerken.

Pupillen gleichweit, reagiren normal. Die Intelligenz vollkommen ungetrübt.

Die Untersuchung der inneren Organe ergiebt keinerlei Abnormität. Die 
Respiration etwas frequent, doch vollständig unbehindert. Urinentleerung geht in durchaus regulärer Weise von Statten. Der Harn ist klar, von hohem. specifischem Gewicht, frei von Albumin und Zucker.

Innerhalb der nächsten 48 Stunden nahm nun die Lähmung der oberen Extremitäten in raschester Weise zu. Am Abend des 8. Juni konnte der Kranke die Arme nicht mehr erbeben, die Ellbogengelenke nur in äusserst beschränktem Grade bewegen, etwas leichter die Finger; doch ermangelten sämmtliche Muskelcontractionen jeglicher Energie. Die rechte Hand war ödematös angeschwollen, über dem dritten Metacarpophalangealgelenk die Hant geröthet und auf Druck schmerzhaft. Röthe und Schwellung am linken jnneren Knöchel dagegen verschwunden.

Am 9. Juni war auch die linise Hand Sitz eines beträchtlichen Oedems, anch hier die Haut über einzelnen Gelenken zwischen Mittelhand and Fingern lebhaft geröthet und empfindlich. Dic Lähmung der Extremitäten hatte weitere Fortschritte gemacht, Patient konnte sich weder im Bett aufrichten, noch seine Lage verändern, Löffel und Glas nicht mehr zum Munde fübren; kurz er befand sich in einem äusserst hülflosen Zustand.

Dagegen war die Sache am 10. Juni ziemlich stationär geworden; eine absolute Lähmung der Arme und Hände war nicht eingetreten, schwache Benge- und Streckbewegungen der Vorderarme, sowie der Finger waren noch mit Anstrengung und ziemlicher Langsamkeit auszuführen.

Die Motilitāt der Fussgelenke und Zehen resp. der Unterschenkelmuskeln war in Verhältniss zu der Unbeweglichkeit der Knie- und Hüftgelenke leidlich conservirt, wenngleich ebenfalls stark herabgesctzt.

Passive Bewegungen liessen sich in allen Gliedern mit der grössten Leichtigkeit ausführen, die paralytischen Muskeln fühlten sich schlaff an: keine Spur von Contracturen.

Das Allgemeinbefinden war trotz des forthestehenden Fiebers (Morgentemperatur bis 38,6 , Abendtemperatur bis 39,2 ) nicht wesentlich gestört; nur der Schlaf war unruhig.

Die Respiration ging vollkommen ungehindert von Statten, ebenso Schlingen und Sprechen, Harn- und Stublentleerung.

Die Stimmung des Kranken war keineswegs deprimirt, sondern neigte sich im Gegentheil zu einem permanenten Optimismus; trotz der fatalen Situation sprach H. immer die Zurersicht aus, dass sich die Sache in kurzer Zeit würde ausgeglichen haben. Uebrigens mussste man nach dem bisherigen Gang der Affection von Stunde zu Stunde den Uebergang der Lähnıng auf lebenswichtige Nerven- und Muskelgebiete befürchten.

Da machte sich im Laufe des 11. Juni in überraschender Weise eine Wiederkelr der Motilität zuerst in den Fingern bemerklich. Während bis jetzt der Druck der Hände fast gleich Null gewesen war, gab derselbe jetzt wenigstens einige Energie kund, die Bewegungsexcursionen der Fingerphalangen waren ausgiebiger geworden. Die unteren Extremitäten zeigten noch keine merkliche Veränderung. Am 12. Juni war auch in letzteren eine Besserung der willkürlichen Contractilität zu constatiren.

Tie Beine konnten nun in den Hüften gebeugt und zu einiger Höhe erhoben werden.

Vorderarm und Hände machten ziemlich ergiebige Bewegungen und 
disponirten über eine relativ beträchtliche Kraft; auch die Schultern wurden nach wiederholten fruchtlosen Versuchen etwas in die Höhe gebracht. Eine Differenz zwischen beiden Körperhälften machte sich zu Gunsten der oberen linken und der unteren rechten Lixtremität geltend.

Am Morgen des 12. Juni war auch die Temperatur normal. Die Oedeme an den Knöcheln hatten sich vollständig verloren, während die Anschwellung der Hände in geringem Grade fortbestand. Die Restitution ging in den folgenden Tagen langsam vorwärts, doch schienen Schwankungen, leichte Rückfälle an einzelnen Tagen dieselbe zu verzögern. So war am 14. Juni die linke Oberextremität wieder mehr paretisch, auf Hand und Vorderarm hatte sich ein starkes Oedem entwickelt. Während an diesem Tage mit Hülfe des kräftiger und mobiler gewordenen rechten Armes das Aufrichten im Bett geJang, batte ein ähnlicher Relaps am 16. diesen Arm heimgesucht. Doch war die Besserung im Ganzen evident.

Dieselbe machte rom 20. bis 29. Juni nun raschere Fortschritte. Die Flexions-, Extensions- und Rotationsbewegungen gingen in allen Gelenken der vier Extremitäten immer kräftiger und prompter, wenn auch unter sichtlich angestrengtem Muskelspiel vor sich.

Am 29. Juni war die letzte Spur des Oedems der rechten Hand verschwunden. Fortdauernd hatte sich die gesteigerte Reflexerregbarkeit in den unteren Extremitäten erhalten und erhielt sich noch längere Zeit in $a b$ nehmendem Grade.

Die Temperatur war des Abends noch leicht erhöht, der Puls stets beschleunigt (bis 140). Eine Functionsstörung von Seiten der vegetativen Organe hatte sich nicht eingestellt, nirgends Deculitusröthe gezeigt.

Am 2. Juli kommt Patient mit Leichtigkeit auf die Füsse zu stehen, am 3. Juli befand er sich einige Zeit ausser Bett, konnte aber noch nicht gehen. Am 4. Juli macht er einige Schritte im Zimmer, fühlt sich aber noch sehr unsicher und bat namentlich in den Wadenmuskeln die Empfindung von Schwäche und Spannung. Am 8. Juli war der Gang mühelos und leicht, kaum schwankend, auch beim Stehen mit gescllossenen Augen tritt nur wenig Schwanken ein. Die Kraft des Händedruckes, der Bewegungen des Vorderarms und der Schultern war im Verhältniss zum Volumen der Muskeln noch vermindert, Gefühl von Schwere und leichter Ermüdung vorhanden, die feinere Disposition der Bewegungen dagegen vollkommen restituirt. Patient schrieb fliessend und kräftig wie vor seiner Erkrankung.

Am 16. Juli war der Gang mit geschlossenen Augen noch recht unsicher, am 18. Juli war auch diese Unsicherheit gewichen. Den 22. Juli. versucht Patient Treppen zu steigen, was ihm ganz gut gelingt. Eine nennenswerthe Abmagerung der betroffenen Muskeln war nicht zu Stande gekommen; abgeseben von einer leichten Abnahme des Fettpolsters hatte sich die Körperfülle des Patienten sehr gut conservirt.

Die - zur Zeit schon eingetretener Besserung vorgenommene - Prüfung der electrischen Erregbarkeit ergab normale Reaction der motorischen Nerven gegen faradischen und galvanischen Strom.

Die Untersuchung wurde nach der von Erb (Archiv für Psychiatrie und Nervenkrankheiten, IV. Bd. 2. Heft) angegebenen Methode angestellt.

Bei der Demonstration des Kranken in der Klinik an 14. Juni war die 
Diagnose auf eine wesentlich in den Vorderhörnern der grauen Rückenmarkssubstanz localisirte entzündliche Affection gestellt worden. Als ätiologisches Moment konnte die Erkältung beim Nachhatsegehen, als prüdisponirend die voransgegangene Excitation des motorischen Apparats beim Tanzen nicht ausgeschlossen werden.

Die Therapie war in den ersten Wochen eine derivatorische (häufge Application von trockenen Schröpflsöpfen zu beiden Seiten der Wirbelsäule und wegen bestehender Obstipation Laxantien).

Vom 24. Juli an wurde zur Tervollständigung der Heilung die Behandlung mit dem galvanischen Strom eingeleitet und bis zum 16. Augnst durchgeführt in folgender Applicationsweise: Strom anfsteigend stabil und Ka labil längs der Wirbelsäule, labile Erregung der Nervenstämme der oberen und unteren Extremitäten : Anode im Nacken, resp. im Kreuz.

Die Behandlung hatte insofern den besten Erfolg, als sich Patient nach den ersten Sitzungen wesentlich kräftiger fühlte; bald konnte derselbe durch weitere hygienische Adjuvantien, Spaziergänge, kalte Bäder die Cur beschleunigen und bei seiner Abreise am 16. August als vollständig genesen betrachtet werden. Kaum erinnert ihn eine leichter eintretende Ermüdung nach grösseren Touren an die vor wenigen Wochen überstandene schwere Alteration des Bewegungsapparates.

Ein Resumé der soeben detaillirten Erscheinungen lässt die hervortretenden Züge des Krankheitsbildes in einer kurzen Skizze markiren.

Ein in der Fülle der Gesundheit stehender junger Mann wird nach einer Erkältung von einer mit Fieber einhergehenden Lähmung zuerst der unteren, bald darauf auch der oberen Extremitäten befallen, die sich durch vorübergehende vage Schmerzen ankündigt. In wenig Tagen hat er die Herrschaft über die Muskeln der Arme und Beine fast complet verloren. Die Sphäre der sensibeln Nerven des Sensoriums und der Intelligenz bleibt vollkommen frei.

Am 12. Tage nach dem Beginn der Erscheinungen wird die Lähmung rückgängig, zuerst in den am spätesten befallenen oberen Extrem:täten, denen bald die unteren in der Retablirung der Motilität folgen. Der Anfangs langsame Gang der Besserung wird von Tag zu Tag ein rascherer; vielleicht beschleunigt durch methodische Galvanisirung erfolgt im Laufe von 10 Wochen - vom Beginn der Affection an gerechnet - vollständige Heilung.

Der geschilderte Fall unterscheidet sich von den Duchenne'schen Typen, wie sie sich in den Beobachtungen ron Cuming, Charcot, Gombault, Bernhardt (Beobachtung 1) und Kussmaul (1. u. 2. Beobachtung) wiederholten, in einigen wesentlichen Zügen. Vor Allem durch die rasche Reparation der Bewegungsstörungen, durch das Ausbleiben atrophischer Zustände der Muskeln, durch das Erhaltensein der faradischen und galvanischen Erregbarkeit. Selbst die 3. Kranke Kussmaul's, die Frey als Beispiel temporärer spinaler Lähmung aufführt, liess noch nach mehreren Monaten Schwäche und Atrophie im linken Deltoides erkennen, die electrische Erregbarkeit gegen beide 
Stromesarten war in zahlreichen Muskelgebieten stark alterirt und kehrte erst nach längerer Zeit (unvollständig) zurück.

Was unseren Fall ausserdem von dem eben erwähnten von Frey unterscheidet, ist die deutlich zu verfolgende progressive Evolution der Lähmung. Gerade hierin scheint mir eine beachtenswerthe Analogie mit der Paralysis ascendens acnta Landry's gegeben. Dass der Gang der Paralyse ein aufsteigender war, darauf lege ich alleidings kein Gewicht, da nicht selten der amgekehrte Modus bei der betreffenden Spinalaffection zu beobachten ist. (Vergl. PellegrinoLévi, Contribution à l'étude de la paralysie ascendante aiguë. Archives générales 1865).

Die Landry'sche Paralyse gilt nun zwar vielfach als eine absolut perniciöse Erkrankung. Selbst Eulenburg (Nervenkrankheiten S. 607) scheint das lethale Ende als characteristisch zu betrachten. Dass diese Auffassung nicht gerechtfertigt ist, dafür bürgen ausser Landry's eigenen Angaben einzelne gut beobachtete Fälle, bei denen auch die schweren "finalen" Symptome, Sprach-, Schling- und Respirationsstörungen, nicht fehlten, zum Beispiel der Fall von $L_{\text {a badi }}$ - L a grave (Gazette des Hòpitanx. 1869. No. 148). -

In der Deutschen Klinik vom Jahre 1871, No. 23, veröffentlicht Reincke unter dem Titel: „ein Fall von Paralysis ascendens acuta" eine Krankengeschichte, welche mit der von uns erzählten grosse Aehnlichkeit darbietet. Die Lähmung entwickelte sich bei Reincke's Patienten, einem 33 jährigen Schiffer, ebenfalls von unten nach oben fortschreitend im Laufe von 14 Tagen, beschränkte sich auf die Extremitäten und blieb nur kurz stationär, um in umgekehrter Reihenfolge sich zurückzubilden und - allerdings nach etwas längerer Zeit - za vollkommener Integrität der Muskelbewegungen zu führen. Die Reflexerregbarkeit war in Reincke's Fall aufgehoben, es bestand starke Hyperästhesie der Muskeln und kam zu hochgradiger Abmagerung der letzteren; ausserdem waren vorübergehendes Oppressionsgefühl und leichte Störungen der Harnentleerung vorhanden. Die beiden von Bernhardt kurz notirten Beobachtungen 2 und 3 scheinen in dieselbe Kategorie zu gehören.

Aus der älteren Literatur führe ich als auffallend analoges Beispiel die Observat. LXXIII in Ollivier's Traité des maladies de la moëlle épinière an.

Man füblt sich unmittelbar versucht, Fälle wie die genannten als früh zum Stillstand kommende, quasi abortive Formen der paralysie ascendante aigue aufzufassen. In der That lässt sich 
begreifen, dass der myelitische Process, der bei der ausgebildeten und ausgeprägten Form unaufhaltsam auf das obere Halsmark und die Medulla oblongata fortschreitet, in den eben characterișirten Fällen rückgängig wird, ohne anf die Centren der Respirations-, Deglntitions-, Articulationsbewegungen überzugreifen. Dass man die anatomischen Veränderungen in dem einen wie in dem anderen Fall als Entzün. dung zu betrachten hat, ist kaum zweifelhaft. Schwerlich wird man versucht sein, auf eine Hyperämie der Medulla (Ollivier u. A.), auf eine einfache Erschöpfung derselben (Jaccoud), eine reflectorische Anämie (Brown-Séquard) zu recurriren oder eine Anomalie. im Wassergehalt der Nervencentra (Pellegrino-Lévi) zu verwuthen.

Duchenne und Joffroy plaidiren bekantlich dafür (und Charcot, Parrot stimmen ihnen bei), dass es sich in einer ganzen Reihe von Lähmungen und Muskelatrophien um eine primitive Alteration der Nervenzellen in den Vorderhörnern handle, - ,altération conduisant à leur atrophie arec tendance à leur disparition." (Vergl. Duchenne et Joffroy: Atrophie des cellules nerveuses de la moëlle et du bulbe, Arch. de physiologie. T. III. 1870).

Der acuten Form dieser Atrophie entspräche die paralysie atrophique de l'enfance und die paralysie antérieure aignë de l'adulte.

Auch bei der paralysis ascendens acuta ist in jüngster Zeit eine herrorstechende Alteration der "motorischen" Zellen gefunden worden. In einem von Chalvet, (Thèse 1871, citirt bei Petitfils p. 95) publicirten Fall ergab die von Kiëner vorgenommene mikroscopische Untersuchung in den Vorderhörnern -des Lendentheils ausser einer Anfüllung der Gefässe und der Maschen des Reticulum der Neuroglia mit einem consistenten Fluidum eine Schwellung und grössere Transparenz der Nervenzellen: „le protoplasma montre des granulations repandues dans un liquide jaunâtre analogue à celui qui remplit les vaisseaux sanguins." Die Veränderung der Ganglienzellen war das wesentliche, Gefässwände und Nervenröhren intact.

Wie man sieht, war es zur Atrophie hier nicht gekommen. Jedenfalls müssen die histologisehen Defecte in vielen Fällen noch geringfügiger sein. Das beweisen die zahlreichen negativen Obductionsbefunde, von denen wenigstens die neueren (z. B. Fall von B as sereau, mikroscopische Untersuchung von Cornil und Ranvier - Petit. fils p. 98; Fall von Bernhardt, Berl. Klin. Wochenschrift 1871. No. 47) kein Misstrauen verdienen.

Andererseits spricht die vollständige und verhältnissmässig rasche Wiederkehr der Function der ergriffenen Nerven- und Muskelgebiete 
in den zur Heilung gelangten Fällen von paralysis ascendens acuta, wie wir oben schon betonten, für die Möglichkeit einer baldigen Reparation der anatomischen Läsion. Letztere mag denn auch in Fällen wie der unsrige eine leichte sein; um eine Atrophie nervöser Appa: rate kann es sich jedenfalls nicht handeln; doch welche histologischen Elemente primitiv oder vorzugsweise ergriffen seien, darüber will ich mir keine hypothetischen Schlüsse erlauben.

Welcher Art nun anch die acute Ernährungsstörung der spinalen Centren gewesen sein mag, die Affection war insofern eine durchaus reine, als sie sich streng anf die motorische Sphäre beschränkte. Abgesehen von vorübergehenden schmerzhaften Sensationen im Beginne traten Sensibilitätsstörungen im ganzen Verlaufe des Falles nicht zn Tage.

Es ist diese strikte Begrenzung der Functionsstörung bei der in Rede stehenden Gruppe von Paralysen nichts weniger als ausnahmslos. Die acute progressive Paralyse sowohl als die paralysie antérieure aiguë de l'adulte $\mathrm{D}$ u chen ne's zeigen häufig mehr weniger tief greifende Beeinträchtigung der Sensibilität. Als Beispiele der ersteren sind in dieser Hinsicht die Fälle von Landry, von Hay em-Oulmont, von Laverau-Bablon, Duchenne's Observat. LXXVI. anzuführen.

Was die acute spinale Lähmung Erwachsener betrifft, so finden sich in einem Fall von Charcot circumscripte. Anästhesien, in einem Fall von Duchenne obtuse Sensibilität in einigen Partien notirt.

Bemerkenswerth ist in dem von uns geschilderten Fall die lange andanernde Steigerug der Reflexerregbarkeit in den unteren Extremitäten, eine Erscheinung, die sich vielleicht durch Ausschaltung des hemmenden Einflusses vom Gehirn aus erklärt.

Dagegen weisen die Oedeme, die circumscripten Erytheme, welche die Invasion und das Höhestadium der Lähmung begleiteten, auf paralytische Zustände der vasomotorischen Nerven oder vasomotorischen Centren (deren Vorhandensein im Rückenmark Goltz sehr wahrscheinlich gemacht hat) hin.

Das Ausbleiben eigentlich trophischer Störungen. war gerade characteristisch und hängt, wie die prompte Wiederkehr der Motilität, mit der vollständigen Reparation der Spinalerkrankung zusammen. Ohne Zweifel sind die Atrophie und Sclerose der Muskeln and Nerven, die Veränderungen des interstitiellen Gewebes mit dem Character chronischer Entzündung und Fettwucherung, die Atrophie der vorderen Spinalwurzeln, die, wie bei der spinalen Kinderlähmung, so anch bei der acnten spinalen Lähmung Erwachsener sich finden, secundärer 
228 Dr. C. Eisenlohr, Zur Lehre von der acuten spinalen Paralyse.

Natur und entsprechen dem chronischen Stadium des myelitischen Torgangs.

In der Gruppe der acuten ascendirenden oder progressiven Paralysen scheinen, soweit die spärlichen Untersuchungen ein Urtheil gestatten, diese Veränderungen der Nerven und Muskeln meist zu fehlen. Doch wurde in einem Fall, bei dem die Entwickelung der Lähmung zu ihrer tödtlichen Höhe längere Zeit in Anspruch nahm, von Hayem in dè Muskelfasern der recti abdominis und der Adductoren des Oberschenkels stellenweise wachsartige Degeneration neben Vermehrung der Muskelkerne nachgewiesen. -

Wenn wir die mitgetheilte Beobachtung mit der acuten aufsteigenden Paralys in Zusammenhang gebracht haben, so geschah dies weniger, um dieselbe mit der Bezeichnung einer bestimmten Kategorie zu versehen, als um durch Hinweisung auf die vielfachen Analogien der genannten noch so räthselhatten Affection mit anderen Spinalerkrankungen, besonders der paralysie antérieure aiguë de l'adulte Duchenne's, den Plan einer einheitlichen Auffassung zo vervollständigen.

So grosse theoretische Bedeutung wir der Aussonderung der Symptomencomplexe, die sich auf die Erkrankung der grauen Vordersäulen des Rückenmarks beziehen, beimessen, müssen wir doch eine scharfe Trennung zur Zeit noch als misslich bezeichnen.

Uebergangsformen zu diffuser Myelitis, welche besonders die Casuistik der paralysis ascendens acnta darbietet, drohen diese von Duchenne so bestimmt gezogene Grenze zwischen Alterationen des vorderen Systems und denjenigen der gesammten grauen Rückenmarkssubstanz zu verwischen.

Die mannigfaltigen Formen der Lähmung wie sie nach acuten Krankheiten rorkommen, bald mit, bald ohne Sensibilitätsstörungen, bald rasch versehwindend, bald unter dem Bilde rapid progressiver Paralyse zum Tode führend, bald von langwieriger Dauer und von atrophischen Zuständen gefolgt - ganz ähnlich denen bei spinaler Kinderlähmung, - schliessen sich offenbar ebenfalls eng an die primitiven Paralysen und Paraplegien, deren Typen sie wiederholen, an. 